\title{
Flowcharts for the diagnosis and treatment of acute cholangitis and cholecystitis: Tokyo Guidelines
}

\author{
Fuminiko Miura ${ }^{1}$, Tadahiro Takada ${ }^{1}$, Yoshifumi Kawarada ${ }^{2}$, Yuji Nimura ${ }^{3}$, Keita Wada ${ }^{1}$, Masahiko Hirota ${ }^{4}$, \\ Masato Nagino $^{3}$, Toshio Tsuyuguchi ${ }^{5}$, Toshihiko Mayumi ${ }^{6}$, Masahiro Yoshida ${ }^{1}$, Steven M. Strasberg ${ }^{7}$, \\ Henry A. Pitt ${ }^{8}$, Jacques Belghiti ${ }^{9}$, Eduardo de Santibanes ${ }^{10}$, Thomas R. Gadacz ${ }^{11}$, Dirk J. Gouma ${ }^{12}$, \\ Sheung-Tat Fan ${ }^{13}$, Mirn-Fu Chen ${ }^{14}$, Robert T. Padbury ${ }^{15}$, Philippus C. Bornman ${ }^{16}$, Sun-Whe Kim ${ }^{17}$, \\ Kui-Hin Liau ${ }^{18}$, Giulio Belli ${ }^{19}$, and Christos Dervenis ${ }^{20}$ \\ ${ }^{1}$ Department of Surgery, Teikyo University School of Medicine, 2-11-1 Kaga, Itabashi-Ku, Tokyo 173-8605, Japan \\ ${ }^{2}$ Mie University School of Medicine, Mie, Japan \\ ${ }^{3}$ Division of Surgical Oncology, Department of Surgery, Nagoya University Graduate School of Medicine, Nagoya, Japan \\ ${ }^{4}$ Department of Gastroenterological Surgery, Kumamoto University Graduate School of Medical Science, Kumamoto, Japan \\ ${ }_{5}^{5}$ Department of Medicine and Clinical Oncology, Graduate School of Medicine, Chiba University, Chiba, Japan \\ ${ }^{6}$ Department of Emergency Medicine and Critical Care, Nagoya University School of Medicine, Nagoya, Japan \\ ${ }^{7}$ Department of Surgery, Washington University in St Louis and Barnes-Jewish Hospital, St Louis, USA \\ ${ }^{8}$ Department of Surgery, Indiana University School of Medicine, Indianapolis, USA \\ ${ }^{9}$ Department of Digestive Surgery and Transplantation, Hospital Beaujon, Clichy, France \\ ${ }^{10}$ Department of Surgery, University of Buenos Aires, Buenos Aires, Argentina \\ ${ }^{11}$ Department of Gastrointestinal Surgery, Medical College of Georgia, Georgia, USA \\ ${ }^{12}$ Department of Surgery, Academic Medical Center, Amsterdam, The Netherlands \\ ${ }^{13}$ Department of Surgery, The University of Hong Kong, Pokfulam, Hong Kong, China \\ ${ }^{14}$ Department of Surgery, Chang Gung Memorial Hospital, Chang Gung University, Taoyuan, Taiwan \\ ${ }^{15}$ Division of Surgical and Specialty Services, Flinders Medical Centre, Adelaide, Australia \\ ${ }^{16}$ Division of General Surgery, University of Cape Town, Cape Town, South Africa \\ ${ }^{17}$ Department of Surgery, Seoul National University College of Medicine, Seoul, Korea \\ ${ }^{18}$ Department of Surgery, Tan Tock Seng Hospital / Hepatobiliary Surgery, Medical Centre, Singapore \\ ${ }^{19}$ Department of General and Hepato-Pancreato-Biliary Surgery, S.M. Loreto Nuovo Hospital, Naples, Italy \\ ${ }^{20}$ First Department of Surgery, Agia Olga Hospital, Athens, Greece
}

\begin{abstract}
Diagnostic and therapeutic strategies for acute biliary inflammation/infection (acute cholangitis and acute cholecystitis), according to severity grade, have not yet been established in the world. Therefore we formulated flowcharts for the management of acute biliary inflammation/infection in accordance with severity grade. For mild (grade I) acute cholangitis, medical treatment may be sufficient/appropriate. For moderate (grade II) acute cholangitis, early biliary drainage should be performed. For severe (grade III) acute cholangitis, appropriate organ support such as ventilatory/circulatory management is required. After hemodynamic stabilization is achieved, urgent endoscopic or percutaneous transhepatic biliary drainage should be performed. For patients with acute cholangitis of any grade of severity, treatment for the underlying etiology, including endoscopic, percutaneous, or surgical treatment should be performed after the patient's general condition has improved. For patients with mild (grade I) cholecystitis, early laparoscopic cholecystectomy is the preferred treatment. For patients with moderate (grade II) acute cholecystitis, early laparoscopic or open cholecystectomy is preferred. In patients with extensive local inflammation, elective cholecystectomy is recommended after initial management with percutaneous gallbladder drainage and/or cholecystostomy. For the patient
\end{abstract}

Offprint requests to: $\mathrm{F}$. Miura

Received: May 31, 2006 / Accepted: August 6, 2006 with severe (grade III) acute cholecystitis, multiorgan support is a critical part of management. Biliary peritonitis due to perforation of the gallbladder is an indication for urgent cholecystectomy and/or drainage. Delayed elective cholecystectomy may be performed after initial treatment with gallbladder drainage and improvement of the patient's general medical condition.

Key words Cholangitis - Acute cholecystitis - Cholecystectomy - Laparoscopic cholecystectomy · Biliary · Drainage · Guidelines

\section{Introduction}

Acute biliary inflammation/infection is classified as either acute cholangitis or acute cholecystitis, and ranges from mild forms that improve with medical treatment to severe forms that require intensive care and urgent intervention. The medical condition of a patient with biliary inflammation/infection is likely to deteriorate rapidly and the condition can become life-threatening. Early diagnosis should be made based on clinical signs/ symptoms and laboratory findings. The type and timing of treatment should be based on the grade of severity of the disease. 


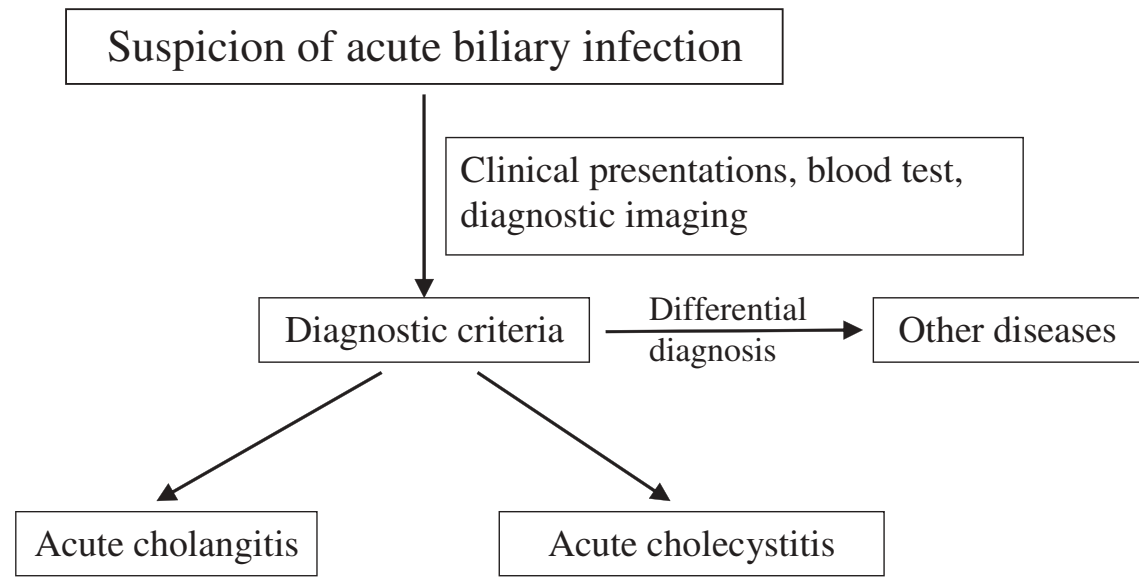

Fig. 1. Flowchart showing general guidance for the management of acute biliary infection
Although endoscopic and laparoscopic techniques have advanced recently (level 1b-2b), ${ }^{1,2}$ the treatment of severe acute biliary inflammation/infection still results in fatalities and increased hospital costs. To our knowledge, there are no definite diagnostic and therapeutic guidelines for acute biliary inflammation/infection according to the grade of severity of the disease. This article describes the management strategy for biliary inflammation/infection in accordance with the severity of the biliary disease. Guidelines were developed, based on best clinical evidence and discussions at the International Consensus Meeting held in Tokyo on April 1-2, 2006.

\section{General guidance for the management of acute biliary inflammation/infection}

A flowchart showing general guidance for the management of acute biliary inflammation/infection is presented in Fig. 1.

\section{Clinical presentation}

Clinical findings associated with acute cholangitis include abdominal pain, jaundice, fever (Charcot's triad), and rigor. The triad was already reported as an indicator of hepatic fever by Charcot in $1877,{ }^{3}$ and has been, historically, used as the generally accepted clinical findings of acute cholangitis. About $50 \%-70 \%$ of patients with acute cholangitis develop all three symptoms (level 2b-4). ${ }^{4-7}$ Reynolds' pentad (Charcot's triad plus shock and a decreased level of consciousness) was presented in 1959, when Reynolds and Dargan ${ }^{8}$ defined acute obstructive cholangitis. The pentad is often used to indicate severe (grade III) cholangitis, but shock and a decreased level of consciousness are observed in only $30 \%$ or fewer patients with acute cholangitis (level $2 \mathrm{~b}-4) \cdot{ }^{4-7}$ A history of biliary disease, such as gallstones, previous biliary procedures, or the placement of a biliary stent are factors that are very helpful to suggest a diagnosis of acute cholangitis.

Clinical symptoms of acute cholecystitis include abdominal pain (right upper abdominal pain), nausea, vomiting, and fever (level 2b-4). ${ }^{9-11}$ The most typical symptom is right epigastric pain. Tenderness in the right upper abdomen, a palpable gallbladder, and Murphy's sign are the characteristic findings of acute cholecystitis. A positive Murphy's sign has a specificity of $79 \%-96 \%$ (level 2b-3b) $)^{9,11}$ for acute cholecystitis.

\section{Blood tests}

The diagnosis of acute cholangitis requires a white blood cell count; measurement of the C-reactive protein level; and liver function tests, including alkaline phosphatase, gamma-glutamyltranspeptidase (GGT), aspartate aminotransferase (AST), alanine aminotransferase (ALT), and bilirubin. Assessment of the severity of the illness requires knowledge of the platelet count, blood urea nitrogen, creatinine, and prothrombin time (PT). Blood cultures are also helpful for severity assessment, as well as for the selection of antimicrobial drugs. Hyperamylasemia is a useful parameter to identify complications such as choledocholithiasis causing biliary pancreatitis (level 1a). ${ }^{12}$

There is no specific blood test for acute cholecystitis; however, the white blood cell count and the measurement of C-reactive protein is very useful in confirming an inflammatory process. Bilirubin, blood urea nitrogen, creatinine, and PT are very useful in assessing the disease severity status of the patient.

\section{Diagnostic imaging}

Abdominal ultrasound (US) and abdominal computerized tomography (CT) with intravenous contrast are very helpful studies in evaluating patients with acute 
biliary tract disease. Abdominal US should be performed in all patients suspected of having acute biliary inflammation/infection. Ultrasound examination has satisfactory diagnostic capability when it is performed not only by specialists but also by emergency physicians (level 1b). ${ }^{13,14}$

The role of diagnostic imaging in acute cholangitis is to determine the presence/absence of biliary obstruction, the level of the obstruction, and the cause of the obstruction, such as gallstones and/or biliary strictures. Assessment should include both US and CT. These studies complement each other and CT may better demonstrate dilatation of the bile duct and pneumobilia.

Some of the characteristic finding of acute cholecystitis include an enlarged gallbladder, thickened gallbladder wall, gallbladder stones and/or debris in the gallbladder, sonographic Murphy's sign, pericholecystic fluid, and pericholecystic abscess. Sonographic Murphy's sign is a very reliable finding of acute cholecystitis, with a specificity exceeding $90 \%$ (level $3 b, 4) .15,16$ CT scan or even plain X-ray may demonstrate free air, pneumobilia, and ileus.

\section{Differential diagnosis}

Diseases which should be differentiated from acute cholangitis are acute cholecystitis, gastric and duodenal ulcer, acute pancreatitis, acute hepatitis, and septicemia of other origins. Diseases which should be differentiated from acute cholecystitis are gastric and duodenal ulcer, hepatitis, pancreatitis, gallbladder cancer, hepatic abscess, Fitz-Hugh-Curtis syndrome, right lower lobar pneumonia, angina pectoris, myocardial infarction, and urinary infection.

\section{Flowchart for the management of acute cholangitis}

A flowchart for the management of acute cholangitis is shown in Fig. 2. The treatment of acute cholangitis should be guided by the grade of severity of the disease. Biliary drainage and antibiotics are the two most important elements of treatment. When a diagnosis of acute cholangitis is suspected, medical treatment, including nil per os (NPO) and the use of intravenous fluids, antibiotics, and analgesia, together with close monitoring of blood pressure, pulse, and urinary output should be initiated. Simultaneously, a severity assessment of the cholangitis should be documented, even if it is mild. Frequent reassessment is important, and patients may need to be reclassified as having mild (grade I), moderate (grade II), or severe (grade III) disease, based on the response to medical treatment. Appropriate treatment should be performed in accordance with the severity grade. Patients with concomitant diseases such as acute pancreatitis or malignant tumor, and elderly patients are likely to progress to a severe level; therefore, such patients should be monitored frequently.

\section{Mild (grade I) acute cholangitis}

Medical treatment may be sufficient. Biliary drainage is not required in most cases. However, for nonresponders to medical treatment, the necessity of biliary

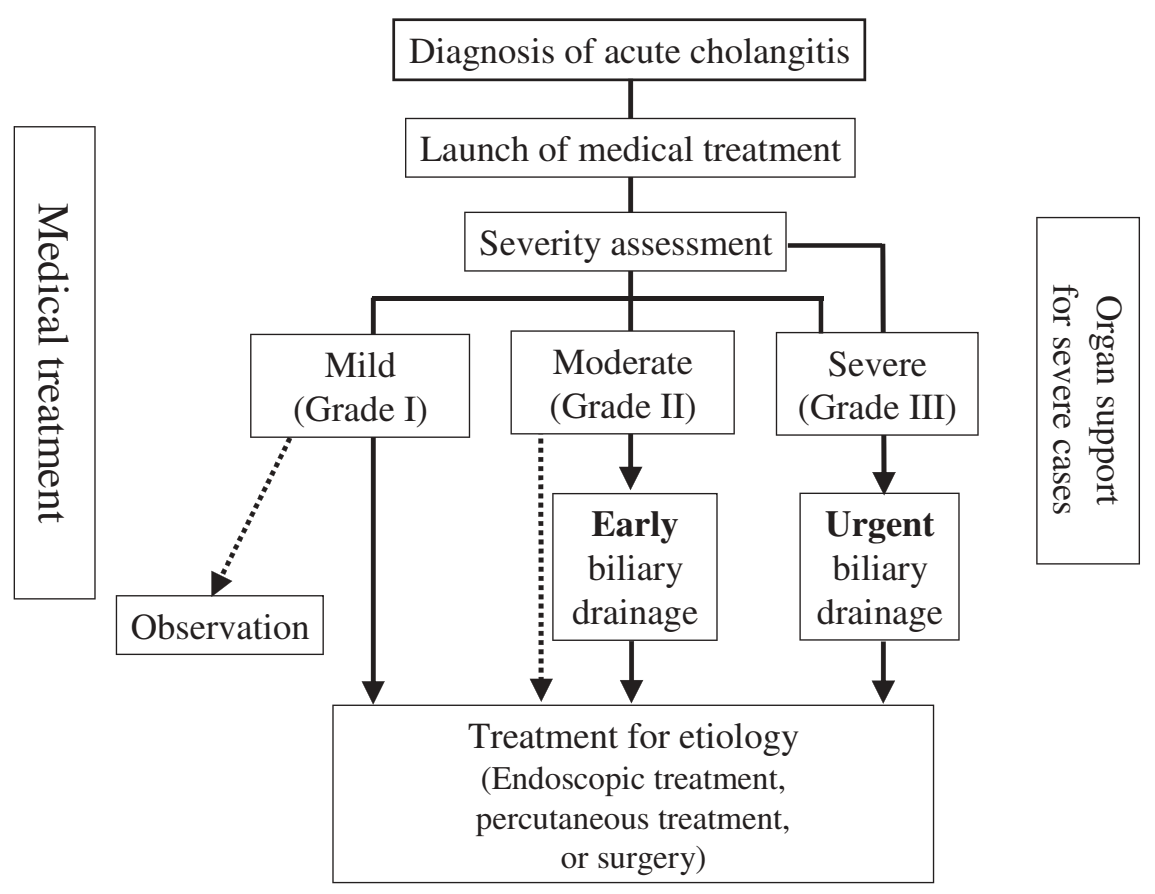

Fig. 2. Flowchart for the management of acute cholangitis 


\section{Panelists $\mathrm{N}=41$}

A

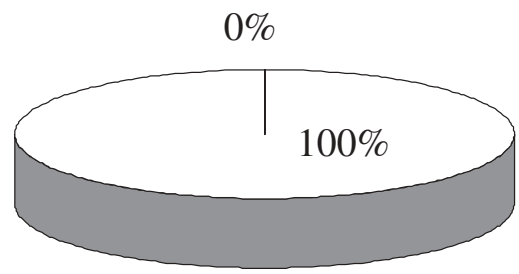

Panelists N=44

B

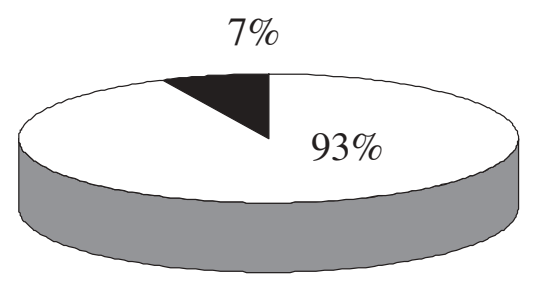

Panelists $N=45$

C

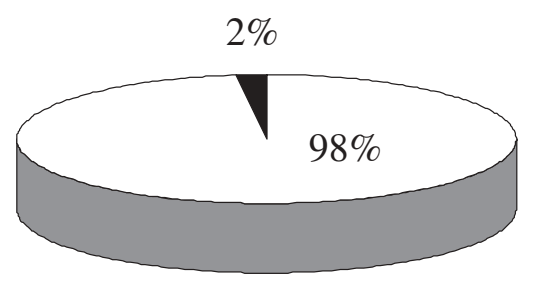

Audience N=67

$\square$ Yes
No

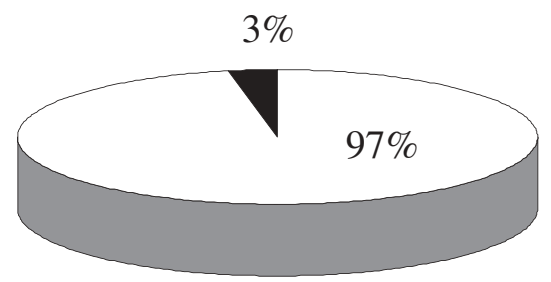

Audience N=67

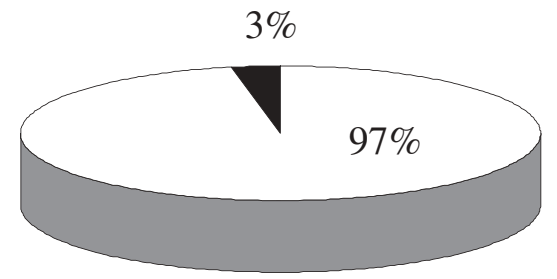

Audience $\mathrm{N}=68$

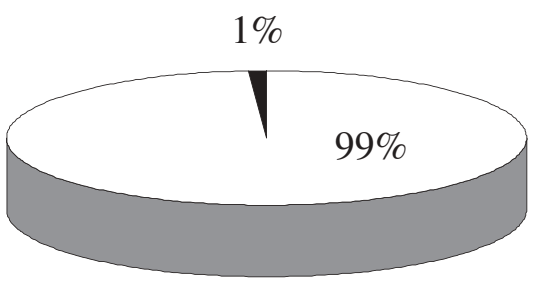

Fig. 3. A Responses to the question "Do you agree with the flowchart for the management of mild acute (grade I) cholangitis?" The flowchart for the management of mild acute (grade I) cholangitis was agreed upon by $100 \%$ and $97 \%$ of the panelists and the audience, respectively. B Responses to the question "Do you agree with the flowchart for the management of moderate acute (grade II) cholangitis?" The flowchart for the management of moderate acute (grade II) cholangitis was agreed upon by $93 \%$ and $97 \%$ of the panelists and the audience, respectively. C Responses to the question "Do you agree with the flowchart for the management of severe acute (grade III) cholangitis?" The flowchart for the management of severe acute (grade III) cholangitis was agreed upon by $98 \%$ and $99 \%$ of the panelists and the audience, respectively drainage should be considered. Treatment options such as endoscopic, percutaneous, or operative intervention may be required, depending on the etiology. Some patients, such as those who develop postoperative cholangitis, may only require antibiotics and generally do not require intervention.

\section{Moderate (grade II) acute cholangitis}

Patients with acute cholangitis who do not respond to medical treatment have moderate (grade II) acute cholangitis. In these patients, early endoscopic or percutaneous drainage or even emergent operative drain- age with a T-tube should be performed. A definitive procedure should be performed to remove the cause of the obstruction once the patient is in a stable condition.

\section{Severe (grade III) acute cholangitis}

Patients with acute cholangitis and organ failure are classified as having severe (grade III) acute cholangitis. These patients require organ support, such as ventilatory/circulatory management (e.g., endotracheal intubation, artificial respiration management, and the use of vasopressin), and treatment for disseminated 


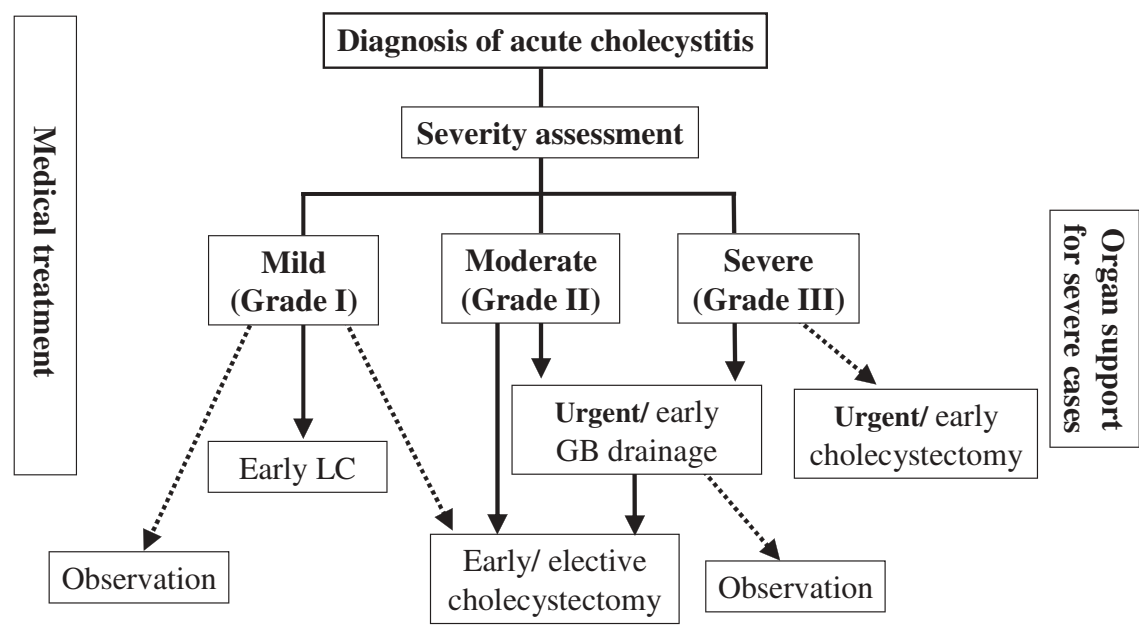

Fig. 4. Flowchart for the management of acute cholecystitis. $G B$, gallbladder; $L C$, laparoscopic cholecystectomy intravascular coagulation (DIC) in addition to the general medical management. Urgent biliary drainage must be anticipated. When the patient is stabilized, urgent (ASAP) endoscopic or percutaneous transhepatic biliary drainage or an emergent operation with decompression of the bile duct with a T-tube should be performed. Definitive treatment of the cause of the obstruction, including endoscopic, percutaneous, or operative intervention, should be considered once the acute illness has resolved.

\section{Results of the Tokyo International Consensus Meeting}

At the International Consensus Meeting, responses to the flowcharts for the management of the different grades of acute cholangitis were elicited and a consensus was reached (Fig. 3).

\section{Flowchart for the management of acute cholecystitis}

A flowchart for the management of acute cholecystitis is shown in Fig. 4. Early cholecystectomy is recommended for most patients, with laparoscopic cholecystectomy as the preferred method. Among high-risk patients, percutaneous gallbladder drainage is an alternative therapy for those patients who cannot safely undergo urgent/ early cholecystectomy (level 4). ${ }^{17,18}$

When a diagnosis of acute cholecystitis is suspected, medical treatment, including NPO, intravenous fluids, antibiotics, and analgesia, together with close monitoring of blood pressure, pulse, and urinary output should be initiated. Simultaneously, the grade of severity needs to be established. Appropriate treatment should be performed in accordance with the severity grade. The assessment of operative risk should also be evaluated based on the severity grade.
After the acute inflammation has been resolved by medical treatment and gallbladder drainage, it is desirable to perform a cholecystectomy to prevent recurrence. In surgically high-risk patients with cholecystolithasis, medical support after percutaneous cholecystolithotomy should be considered (level 4). ${ }^{19-21}$ For patients with acalculous cholecystitis, cholecystectomy is not required, because recurrence of acute acalculous cholecystitis after gallbladder drainage is rare (level 4)..$^{17,22}$

\section{Mild (grade I) acute cholecystitis}

Early laparoscopic cholecystectomy is the preferred treatment. Elective cholecystectomy may be selected (if early cholecystectomy is not performed) in order to improve other medical problems.

\section{Moderate (grade II) acute cholecystitis}

Early laparoscopic or open cholecystectomy is preferred. If a patient has serious local inflammation making early cholecystectomy difficult, then percutaneous or operative drainage of the gallbladder is recommended. Elective cholecystectomy can be performed after improvement of the acute inflammatory process.

\section{Severe (grade III) acute cholecystitis}

Severe (grade III) acute cholecystitis is accompanied by organ dysfunction and/or severe local inflammation. Appropriate organ support in addition to medical treatment is necessary for patients with organ dysfunction. Management of severe local inflammation by percutaneous gallbladder drainage and/or cholecystectomy is needed. Biliary peritonitis due to perforation of the gallbladder is an indication for urgent cholecystectomy 
Panelists N=39

A

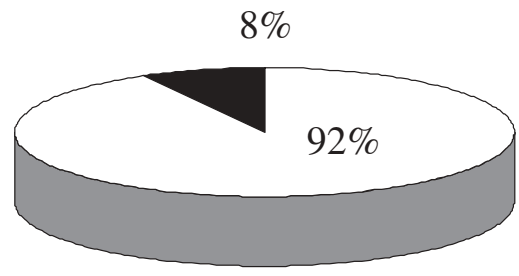

Japanese panelists $\mathrm{N}=19$

B

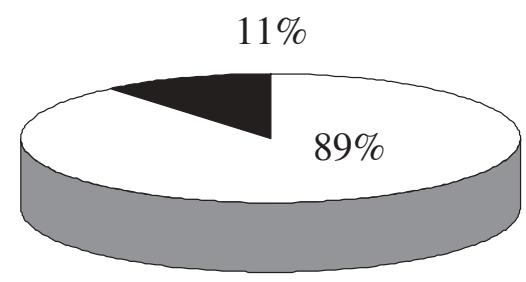

Panelists $\mathrm{N}=39$

C

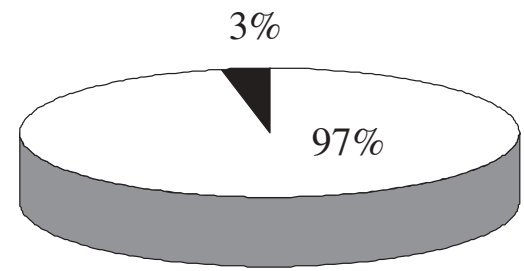

Audience N=63

$\square$ Yes
No

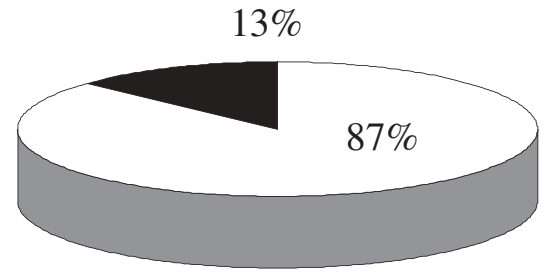

Japanese audience $\mathrm{N}=65$

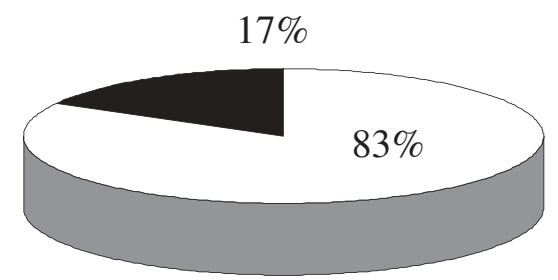

Audience N=66

Fig. 5. A Responses to the question "Do you agree with the flowchart for the management of mild acute (grade I) cholecystitis?" The flowchart for the management of mild acute (grade I) cholecystitis was agreed upon by $92 \%$ and $87 \%$ of the panelists and the audience, respectively. B Responses to the question "Do you agree with the flowchart for the management of moderate acute (grade II) cholecystitis?" The flowchart for the management of moderate acute (grade II) cholecystitis was agreed upon by $89 \%$ and $83 \%$ of the Japanese panelists and the Japanese audience, respectively. C Responses to the question "Do you agree with the flowchart for the management of severe acute (grade III) cholecystitis?" The flowchart for the management of severe acute (grade III) cholecystitis was agreed upon by $97 \%$ and $95 \%$ of the panelists and audience, respectively

and drainage. Elective cholecystectomy may be performed after improvement of the acute illness by gallbladder drainage.

\section{Results of the Tokyo International Consensus Meeting}

At the International Consensus Meeting, flowcharts for the management of mild (grade I) and severe (grade III) acute cholecystitis were agreed upon by almost all of the participants; however, the flowchart for moderate (grade II) acute cholecystitis was agreed upon by fewer than $90 \%$ of the participants (Fig. 5).
Acknowledgments. We would like to express our deep gratitude to the Japanese Society for Abdominal Emergency Medicine, the Japan Biliary Association, and the Japanese Society of Hepato-Biliary-Pancreatic Surgery, who provided us with great support and guidance in the preparation of the Guidelines. This process was conducted as part of the project for the Preparation and Diffusion of Guidelines for the Management of Acute Cholangitis (H-15-Medicine-30), with a research subsidy for fiscal 2003 and 2004 (Integrated Research Project for Assessing Medical Technology), sponsored by the Japanese Ministry of Health, Labour, and Welfare. 
We also truly appreciate the panelists who cooperated with and contributed significantly to the International Consensus Meeting held in Tokyo on April 1 and 2, 2006.

\section{References}

1. Lai EC, Mok FP, Tan ES, Lo CM, Fan ST, You KT, et al. Endoscopic biliary drainage for severe acute cholangitis. N Engl J Med 1992;326:1582-6. (level 2b)

2. Lo CM, Liu CL, Fan ST, Lai EC, Wong J. Prospective randomized study of early versus delayed laparoscopic cholecystectomy for acute cholecystitis. Ann Surg 1998;227:461-7. (level 1b)

3. Charcot M. De la fievre hepatique symptomatique Comparison avec la fievre uroseptique. Paris: Bourneville et Sevestre, 1877. (level 4)

4. Boey JH, Way LW. Acute cholangitis. Ann Surg 1980;191:264-70. (level 4)

5. Csendes A, Diaz JC, Burdiles P, Maluenda F, Morales E. Risk factors and classification of acute suppurative cholangitis. $\mathrm{Br} \mathrm{J}$ Surg 1992;79:655-8. (level 2b)

6. Welch JP, Donaldson GA. The urgency of diagnosis and surgical treatment of acute suppurative cholangitis. Am J Surg 1976; 131:527-32. (level 4)

7. O'Connor MJ, Schwartz ML, McQuarrie DG, Sumer HW. Acute bacterial cholangitis: an analysis of clinical manifestation. Arch Surg 1982;117:437-41. (level 4)

8. Reynolds BM, Dargan EL. Acute obstructive cholangitis; a distinct clinical syndrome. Ann Surg 1959;150:299-303. (level 4)

9. Eskelinen M, Ikonen J, Lipponen P. Diagnostic approaches in acute cholecystitis; a prospective study of 1333 patients with acute abdominal pain. Theor Surg 1993;8:15-20. (level 2b)

10. Staniland JR, Ditchburn J, De Dombal FT. Clinical presentation of acute abdomen: study of 600 patients. BMJ 1972;3:393-8. (level 4)

11. Trowbridge RL, Rutkowski NK, Shojania KG. Does this patient have acute cholecystitis? JAMA 2003;289:80-6. (level 3b)

12. Abboud PA, Malet PF, Berlin JA, Staroscik R, Cabana MD, Clarke JR, et al. Predictors of common bile duct stones prior to cholecystectomy: a meta-analysis. Gastrointest Endosc 1996;44:450-5. (level 1a)

13. Rosen CL, Brown DF, Chang Y, Moore C, Averill NJ, Arkoff LJ, et al. Ultrasonography by emergency physicians in patients with suspected cholecystitis. Am J Emerg Med 2001;19:32-6. (level 1b)

14. Kendall JL, Shimp RJ. Performance and interpretation of focused right upper quadrant ultrasound by emergency physicians. J Emerg Med 2001;21:7-13. (level 1b)

15. Ralls PW, Halls J, Lapin SA, Quinn MF, Morris UL, Boswell W. Prospective evaluation of the sonographic Murphy sign in suspected acute cholecystitis. J Clin Ultrasound 1982;10:113-5. (level 4)

16. Soyer P, Brouland JP, Boudiaf M, Kardache M, Pelage JP, Panis Y, et al. Color velocity imaging and power Doppler sonography of the gallbladder wall: a new look at sonographic diagnosis of acute cholecystitis. Am J Roentgenol AJR 1998;171:183-8. (level 3b)

17. Sugiyama M, Tokuhara M, Atomi Y. Is percutaneous cholecystostomy the optimal treatment for acute cholecystitis in the very elderly? World J Surg 1998;22:459-63. (level 4)

18. Chopra S, Dodd GD 3rd, Mumbower AL, Chintapalli KN, Schwesinger WH, Sirinek KR, et al. Treatment of acute cholecystitis in non-critically ill patients at high surgical risk: comparison of clinical outcomes after gallbladder aspiration and after percutaneous cholecystostomy. Am J Roentgenol AJR 2001;176:102531. (level 4)
19. Inui K, Nakazawa S, Naito Y, Kimoto E, Yamao K. Nonsurgical treatment of cholecystolithiasis with percutaneous transhepatic cholecystoscopy. Am J Gastroenterol 1988;83:1124-7. (level 4)

20. Boland GW, Lee MJ, Mueller PR, Dawson SL, Gaa J, Lu DS, et al. Gallstones in critically ill patients with acute calculous cholecystitis treated by percutaneous cholecystostomy: nonsurgical therapeutic options. Am J Roentgenol AJR 1994;162:1101-3. (level 4)

21. Majeed AW, Reed MW, Ross B, Peacock J, Johnson AG. Gallstone removal with a modified cholecystoscope: an alternative to cholecystectomy in the high-risk patient. J Am Coll Surg 1997;184:27380. (level 4)

22. Shirai Y, Tsukada K, Kawaguchi H, Ohtani T, Muto T, Hatakeyama K. Percutaneous transhepatic cholecystostomy for acute acalculous cholecystitis. Br J Surg 1993;80:1440-2. (level 4)

\section{Discussion at the Tokyo International Consensus Meeting}

\section{General guidance}

Acute biliary inflammation/infection consists of acute cholangitis and acute cholecystitis. In these infectious diseases, bacterial contamination is an essential condition, but inflammation has a wider meaning and includes not only infection but also other inflammation caused by non-bacterial vectors (Sun-Whe Kim, Korea). It may be difficult to initially determine whether the inflammation is progressing to an bacterial infection (Thomas $\mathrm{R}$. Gadacz, USA); therefore, in this article, we adopted the term "acute biliary inflammation/infection".

As for general guidance for the management of acute biliary inflammation/infection, most aspects were accepted with great concordance. During the initial evaluation of a patient, information on a past history of biliary disease (gallstone, previous biliary surgery, and biliary stent placement) was emphasized (Jacques Belghiti, France; Philippus C. Bornman, South Africa; and Steven M. Strasberg, USA). Jacques Belghiti added that septicemia arising from other diseases needs to be differentiated from acute cholangitis.

\section{Flowchart for the management of acute cholangitis}

Concerning the treatment of acute cholangitis, the particular importance of antibiotics as well as urgent biliary drainage was confirmed (Jacques Belghiti; Joseph W.Y. Lau, Hong Kong, and Steven M. Strasberg). There were few controversial matters in the flowchart for the management of acute cholangitis. Joseph W.Y. Lau advocated that mild cholangitis and moderate cholangitis should be combined, because many patients with moderate cholangitis would easily revert to the mild grade within $12 \mathrm{~h}$ after successful medical treatment, and he suggested that severity assessment should depend on whether patients responded to the initial treatment. This statement implies that severity assessment should be 
repeated after the initiation of treatment for acute cholangitis.

\section{Flowchart for the management of acute cholecystitis}

There were several controversies over the treatment of acute cholecystitis. Early cholecystectomy is indicated for most patients with acute cholecystitis, and laparoscopic cholecystectomy is preferred for experienced surgeons. Several randomized controlled trials comparing early and delayed operation conducted in the 1970s to 1980 s found that early surgery had the advantages of less blood loss, shorter operation time, a lower complication rate, and a shorter hospital stay. Some Japanese doctors advocated that early cholecystectomy should not be recommended because early cholecystectomy was not prevalent in Japan. Steven M. Strasberg mentioned: "We have to be willing to accept the fact that we may need to change our practice based upon the evidence". Results of randomized controlled trials comparing early laparoscopic cholecystectomy with delayed laparoscopic cholecystectomy have also shown that early laparoscopic surgery is superior to delayed surgery in terms of the conversion rate to open surgery, complication rate, and total hospital stay. Toshihiko Mayumi (Japan) mentioned that because laparoscopic cholecystectomy by inexperienced surgeons resulted in more frequent intraoperative complications than open cholecystectomy, the laparoscopic procedure should not be overemphasized.

There was more discussion to determine the treatment strategy for acute moderate (grade II) cholecysti- tis. Before the start of the international symposium it was considered that urgent/early cholecystectomy should be performed for these patients. Steven M. Strasberg mentioned: "For patients with acute moderate cholecystitis (patients who have a white [cell] count over 18000; patients who have cholecystitis for more than $72 \mathrm{~h}$; patients who have a palpable inflammatory mass), early cholecystectomy is going to be maybe very difficult. Therefore do we really want to say to the general surgeon in a small hospital that we recommend that when the white [cell] count is over 18000 that he takes the patient to the operating room? I do not think so." After the statement of his opinion, delayed elective cholecystectomy was recommended for acute moderate (grade II) cholecystitis with severe local inflammation. On the other hand, Eduardo de Santibanes (Argentina) advocated that early laparoscopic cholecystectomy could be performed for patients with acute moderate cholecystitis.

The treatment courses for mild (grade I) and severe (grade III) cholecystitis were accepted without major adverse opinions. The recommendation of early laparoscopic cholecystectomy for mild (grade I) cases and gallbladder drainage for severe (grade III) cases obtained consensus. Some Japanese doctors suggested that endoscopic gallbladder drainage as well as percutaneous gallbladder drainage should be recommended. However, Jacques Belghiti rejected this suggestion, because there was poor evidence for efficacy, and because endoscopic gallbladder drainage needed a special technique. Thomas R. Gadacz added surgical cholecystostomy to one of the methods for gallbladder drainage. 